\title{
A portable cryostat for the cold transfer of polarized solid HD targets: HDice-I
}

\author{
C.D. Bass ${ }^{\mathrm{a}, 1}$, C. Bade ${ }^{\mathrm{g}}$, M. Blecher ${ }^{\mathrm{n}}$, A. Caracappa ${ }^{\mathrm{b}}$, A. D'Angelo $^{\mathrm{k}, \mathrm{a}}$, A. Deur ${ }^{\mathrm{a}}$, G. Dezern ${ }^{\mathrm{a}}$, \\ H. Glueckler ${ }^{c}$, C. Hanretty ${ }^{\mathrm{m}}$, D. Ho ${ }^{\mathrm{d}}$, A. Honig ${ }^{\mathrm{h}, 2}$, T. Kageya ${ }^{\mathrm{a}, \mathrm{n}}$, M. Khandaker ${ }^{\mathrm{f}}$, V. Laine ${ }^{\mathrm{a}, \mathrm{i}}$, \\ F. Lincoln ${ }^{\mathrm{b}}$, M.M. Lowry ${ }^{\mathrm{a}, \mathrm{b}}$, J.C. Mahon ${ }^{\mathrm{g}}$, T. O'Connell ${ }^{\mathrm{j}}$, M. Pap ${ }^{\mathrm{c}}$, P. Peng ${ }^{\mathrm{m}}$, B. Preedom ${ }^{\mathrm{l}}$, \\ A.M. Sandorfi ${ }^{\mathrm{a}, \mathrm{b}, *}$, H. Seyfarth ${ }^{\mathrm{c}}$, H. Stroeher ${ }^{\mathrm{c}}$, C.E. Thorn ${ }^{\mathrm{b}}$, X. Wei $^{\mathrm{a}, \mathrm{b}}$, C.S. Whisnant $^{\mathrm{e}}$ \\ a Thomas Jefferson National Accelerator Facility, Newport News, VA 23606, USA \\ ${ }^{\mathrm{b}}$ Brookhaven National Laboratory, Upton, NY 11973, USA \\ c Forschungszentrum Jülich GmbH, Jülich, Germany \\ ${ }^{\mathrm{d}}$ Carnegie-Mellon University, Pittsburgh, PA 15213, USA \\ e James Madison University, Harrisonburg, VA 22807, USA \\ ${ }^{\mathrm{f}}$ Norfolk State University, Norfolk, VA 23504, USA \\ g Ohio University, Athens, $\mathrm{OH} 45701$, USA \\ h Syracuse University, Syracuse, NY 13210, USA \\ ${ }^{\mathrm{i}}$ Université Blaise Pascal, Clermont-Ferrand, 63177 Aubiere, France \\ ${ }^{\mathrm{j}}$ University of Connecticut, Storrs-Mansfield, CT 06269, USA \\ k Universita' di Roma “Tor Vergata" and INFN Sezione di Roma2, 00133 Roma, Italy \\ ${ }^{1}$ University of South Carolina, Columbia, SC 29208, USA \\ ${ }^{\mathrm{m}}$ University of Virginia, Charlottesville, VA 22903, USA \\ ${ }^{n}$ Virginia Polytechnic Institute and State University, Blacksburg, VA 24061, USA
}

\section{A R T I C L E I N F O}

\section{Article history:}

Received 17 September 2013

Received in revised form

23 October 2013

Accepted 24 October 2013

Available online 31 October 2013

Keywords:

Solid hydrogen deuteride

Frozen-spin target

Spin relaxation

Halbach rare-earth magnet

\begin{abstract}
A B S T R A C T
A device has been developed with moveable liquid nitrogen and liquid helium volumes that is capable of reaching over $2 \mathrm{~m}$ into the coldest regions of a cryostat or dilution refrigerator and reliably extracting or installing a target of solid, polarized hydrogen deuteride (HD). This Transfer Cryostat incorporates a cylindrical neodymium rare-earth magnet that is configured as a Halbach dipole, which is maintained at $77 \mathrm{~K}$ and produces a $0.1 \mathrm{~T}$ field around the HD target. Multiple layers provide a hermetic $77 \mathrm{~K}$-shield as the device is used to maintain a target at $2 \mathrm{~K}$ during a transfer between cryostats. Tests with frozen-spin HD show very little polarization loss for either $\mathrm{H}(-1 \pm 2 \%$, relative) or $\mathrm{D}(0 \pm 3 \%$, relative $)$ over typical transfer periods. Multiple target transfers with this apparatus have shown an overall reliability of about 95\% per transfer, which is a significant improvement over earlier versions of the device.
\end{abstract}

(c) 2013 Elsevier B.V. All rights reserved.

\section{Introduction}

A successful description of the excitation and decay modes of a composite system is a basic test of how well the underlying forces are understood. Quantum-Chromodynamics (QCD) has been able to account for many of the properties of hadrons, although the excited states of the nucleon continue to pose significant challenges. These excited states are short-lived, and only the lowest-energy nucleon excitation - the $\Delta(1232)$ resonance - is

\footnotetext{
* Corresponding author. Tel.: + 1 7572695457; fax: +1 7572696418 .

E-mail addresses: bassc@lemoyne.edu (C.D. Bass),

sandorfi@JLab.org (A.M. Sandorfi).

1 Current address: Le Moyne College, Syracuse, NY 13214, USA.

2 Deceased.
}

moderately well-isolated. Higher-energy excitations appear as broad and overlapping resonances in pion scattering and meson photoproduction, which can only be disentangled through the application of detailed partial wave analysis (PWA) techniques. Furthermore, many more excited states have been predicted $[1,2]$ than have been extracted using PWA.

A number of factors complicate the study of excited nucleon states [3]. Most important is the lack of experimental data on the majority of the 16 possible spin matrix elements in pseudoscalar meson photoproduction, which has created ambiguities in PWA [4]. To address this shortcoming, experiments have been conducted with frozen-spin targets of solid hydrogen deuteride (HD) at the Brookhaven National Laboratory (BNL) in the $\Delta(1232)$ resonance region [5], and at the Thomas Jefferson National Accelerator Facility (JLab) throughout the higher-energy resonance 
excitation region [6]. The goal of the latter has been a complete experiment, in which most (if not all) possible reaction observables involving explicit spin orientations of the beam, target, and recoiling baryon are measured. More than half of the spin observables cannot be determined without a polarized target, and the use of a polarized target in conjunction with measurements of double- and triple-polarization asymmetries can determine all 16 of them with a single orientation of target polarization [4].

As a polarized target for nuclear and particle physics, frozenspin HD (known locally as HDice) has many attractive features. Apart from minimal contributions from the target cell, the HD material is low-Z, pure, and free of background sources. In addition, HD contains a single polarizable neutron and this freedom from dilution gives HD experiments focusing on reactions with polarized neutrons, such as JLab E06-101, a particularly high figure of merit [6]. However, the production of frozen-spin HD is considerably more complex than that of conventional polarized nuclear targets, requiring several specially designed and constructed cryostats and target manipulation tools, as well as a number of commercial dewars and dilution refrigerator and magnet systems.

This is the first of a series of papers that describe the preparation, polarization, and handling of frozen-spin polarized HD targets. The ability to move these targets between cryostats without significant polarization loss is crucial because the targets are produced and polarized in a location separated from the experimental hall and are transported to a detector once they have reached a frozen-spin state. This paper describes the design and operation of a Transfer Cryostat (TC) that has been used at BNL and JLab for moving HD targets between cryostats. These operations are complex and typically require several hours. The TC discussed here is the current and most successful design of a series of devices that evolved during the HD target program. (Condensed discussions of earlier designs can be found in Refs. [7-9]).

\section{The HD target production and run sequence}

We begin with a brief description of the typical sequence used to create a frozen-spin polarized HD target and transport it to an In-Beam Cryostat located in an experimental hall for use in nuclear physics experiments. The HD target production requirements and cold transport steps have determined the TC design characteristics.

\subsection{Production of a HD target}

An empty target cell (Fig. 1) is mounted on the end of a capillary tool and lowered into the cold-bore of a variabletemperature cryostat referred to as the Production Dewar (PD). The target cell is composed of a shell of polychlorotrifluoroethylene $^{3}$ (pCTFE) that is fixed to a copper target ring possessing an interior left-handed (LH) M26 $\times 1$ thread and exterior righthanded $(\mathrm{RH}) \mathrm{M} 35 \times 1$ thread. Thin aluminum cooling wires span the interior of the pCTFE shell and are soldered into the target ring (800-2100 in number, depending on the diameter of the target cell).

The target cell is cooled to just above the triple-point temperature of HD (16.6 K), and distilled HD gas is condensed through the capillary and into the target cell. The HD gas contains trace amounts of $\mathrm{H}_{2}$ and $\mathrm{D}_{2}$ that are necessary for the polarization process [9]. Once the target cell has been filled with liquid HD, the temperature is lowered, creating a solid HD crystal. The cell is then

\footnotetext{
${ }^{3}$ The chemical composition for polychlorotrifluoroethylene is $\mathrm{C}_{2} \mathrm{ClF}_{3}$.
}

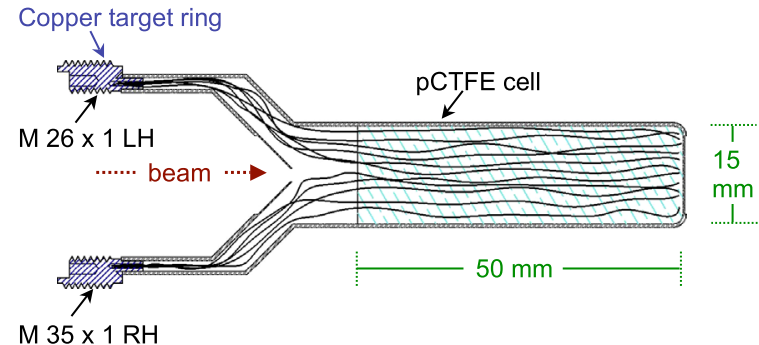

Fig. 1. Cross-section of a typical HD target that was used during the E06-101 experiment at JLab. A shell of pCTFE is attached to a copper target ring that has an interior left-handed thread (engaged by tools to move the target cell) and exterior right-handed thread (used to mount the target in a dewar). Several hundred thin aluminum wires span the length of the target cell and are soldered at one end into the target ring. A $50 \mathrm{~mm}$ long cylindrical crystal of HD is grown in the target cell by condensing and then freezing distilled HD gas. The embedded aluminum cooling wires allow heat, which is generated in the HD crystal during the polarization process and in-beam experiments, to escape from the target and into the mixing chamber of a dilution fridge.

lowered to the $4 \mathrm{~K}$ copper threads of the PD where the capillary tool is removed.

The HD target is then positioned within a set of crossed coils housed inside the PD that are used for NMR studies [10]. The polarizations $P(t)$ for $\mathrm{H}$ and for $\mathrm{D}$ in the target increase to their thermal equilibrium values (as determined by the PD field and temperature) with a time dependence parameterized as

$P(t)=P_{T E}\left(1-e^{-t / T_{1}}\right)$

with time $t$, equilibrium polarization $P_{T E}$, and relaxation time $T_{1}$. At this stage the relaxation time is of the order of seconds for $\mathrm{H}$ and $10 \mathrm{~s}$ to $100 \mathrm{~s}$ of seconds for $\mathrm{D}$, depending on the concentrations of the $\mathrm{H}_{2}$ and $\mathrm{D}_{2}$ impurities as well as the temperature and field strength in the PD; consequently, the $\mathrm{H}$ and $\mathrm{D}$ quickly reach their respective thermal equilibrium polarizations. These polarization levels are small (less than 0.001 under PD conditions) but are more than adequate to calibrate the NMR electronic circuit.

\subsection{Polarizing the HD target}

The HD target is extracted from the PD with the use of the TC, which has a liquid helium (LHe) volume that can rotate and translate within the TC (Section 3.1) and extend into the cold bore of another cryostat. The LHe volume incorporates a M26 $\times 1 \mathrm{LH}$ thread on its lower end that matches the interior thread of the target ring. The LHe volume is extended into the PD until it contacts the target, after which counterclockwise rotation of the LHe volume screws the TC thread into the HD target cell until it is fully engaged. Continued counterclockwise rotation of the LHe volume causes the exterior M35 $\times 1 \mathrm{RH}$ thread on the HD target ring to unscrew from the cold-bore of the PD. The LHe volume and attached target are withdrawn into the TC, which is then moved from the PD to an Oxford-1000 dilution refrigerator and superconducting magnet system (DF). The transfer operation is shown schematically in Fig. 2. The PD and DF are arranged in pits so that their attachment points are at a common level.

Once attached to the DF, the TC LHe volume and HD target are extended and rotated clockwise to engage the exterior thread of the target ring into the matching thread in the cold-bore of the DF. Once the HD target is fully engaged in the cold-bore, continued rotation unscrews the thread on the end of the TC LHe volume from the target ring, and the LHe volume is then withdrawn into the TC. The dimensions of the TC are constrained by the narrow cross-section and depth of the cold-bore and polarizing magnets within the DF. 
After the HD target is secured within the DF, the field is raised to $15 \mathrm{~T}$ and the temperature lowered to the $15 \mathrm{mK}$ region. Because of wavefunction symmetry requirements, the first rotational states of the $\mathrm{H}_{2}$ and $\mathrm{D}_{2}$ impurities present in the HD are polarizable and metastable. These polarize rapidly and transfer their polarization to $\mathrm{HD}$, leading to typical values in the range $\mathrm{P}(\mathrm{H}) \sim 60 \pm 5 \%$ and $\mathrm{P}(\mathrm{D}) \sim 18 \pm 5 \%$. As the metastable impurities decay away (with relaxation times of 6 and 18 days for $\mathrm{H}_{2}$ and $\mathrm{D}_{2}$, respectively), the solid HD reaches a frozen-spin state [9]. A target is nominally held in the DF at high field and low temperature for approximately 2-3 months to allow the spins of the $\mathrm{H}$ and the $\mathrm{D}$ to decouple from the HD lattice.

\subsection{Transporting the HD target to the experimental hall}

The HD target is transferred from the DF and back into the PD for evaluation of its polarization using NMR. For this transfer, the TC must provide both a holding field as well as low temperature.

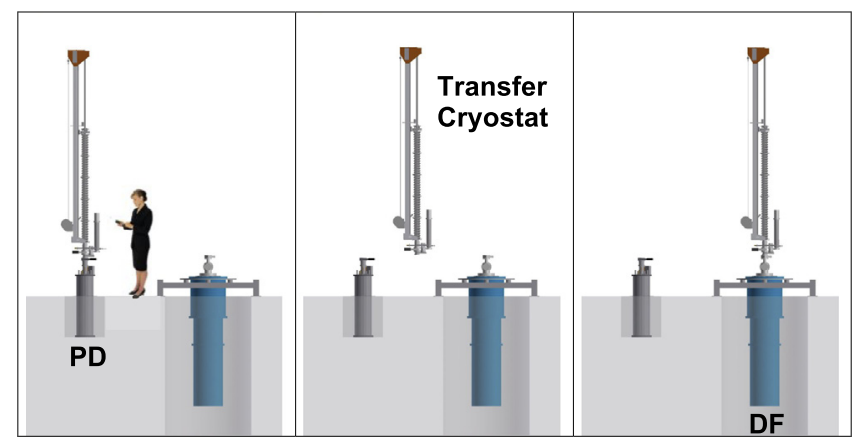

Fig. 2. Schematic of a typical cryostat arrangement during the stages of an HD transfer. Cryostats used in the polarization cycle are positioned in pits so that their TC attachment points are at a common level.
While relaxation times of months to years are easily reached at high field and very low temperatures, the size constraints of the TC limit the strength of the transport field and the operational temperature. This results in target transfers where the HD experiences significantly shorter relaxation times that vary approximately with $B / T$ (on the order of a day for $P_{H}$ and a week for $P_{D}$ under TC conditions).

The HD target is transferred to a Storage Dewar (SD) and held at $1.6 \mathrm{~K}$ and $5 \mathrm{~T}$ until it is needed for a nuclear physics experiment. The SD and TC are subsequently moved to the experimental area. From a staging area within the experimental hall, the HD target is extracted from the SD and loaded into the In-Beam Cryostat (IBC) for the experiment.

\subsection{Post-experiment calibrations}

The IBC contains an NMR polarimeter that provides frequent measurements during an experiment. This is cross-calibrated with the polarimeter of the PD where the initial thermal equilibrium polarization measurements have been performed. Transfer losses have been estimated by carrying out multiple transfers between the PD and the IBC. As a final cross-calibration after the experimental run, the HD target is transferred from the IBC to the PD (within the experimental hall) for a final polarization measurement and then evaporated for gas analysis.

\section{Design and construction features}

The cold transfer of a target between cryostats with the TC is accomplished by engaging and rotating the threads of the target ring (Fig. 1), as discussed above. A target can be unscrewed from one cryostat, withdrawn into the TC, and then inserted and screwed into another cryostat. During transport between
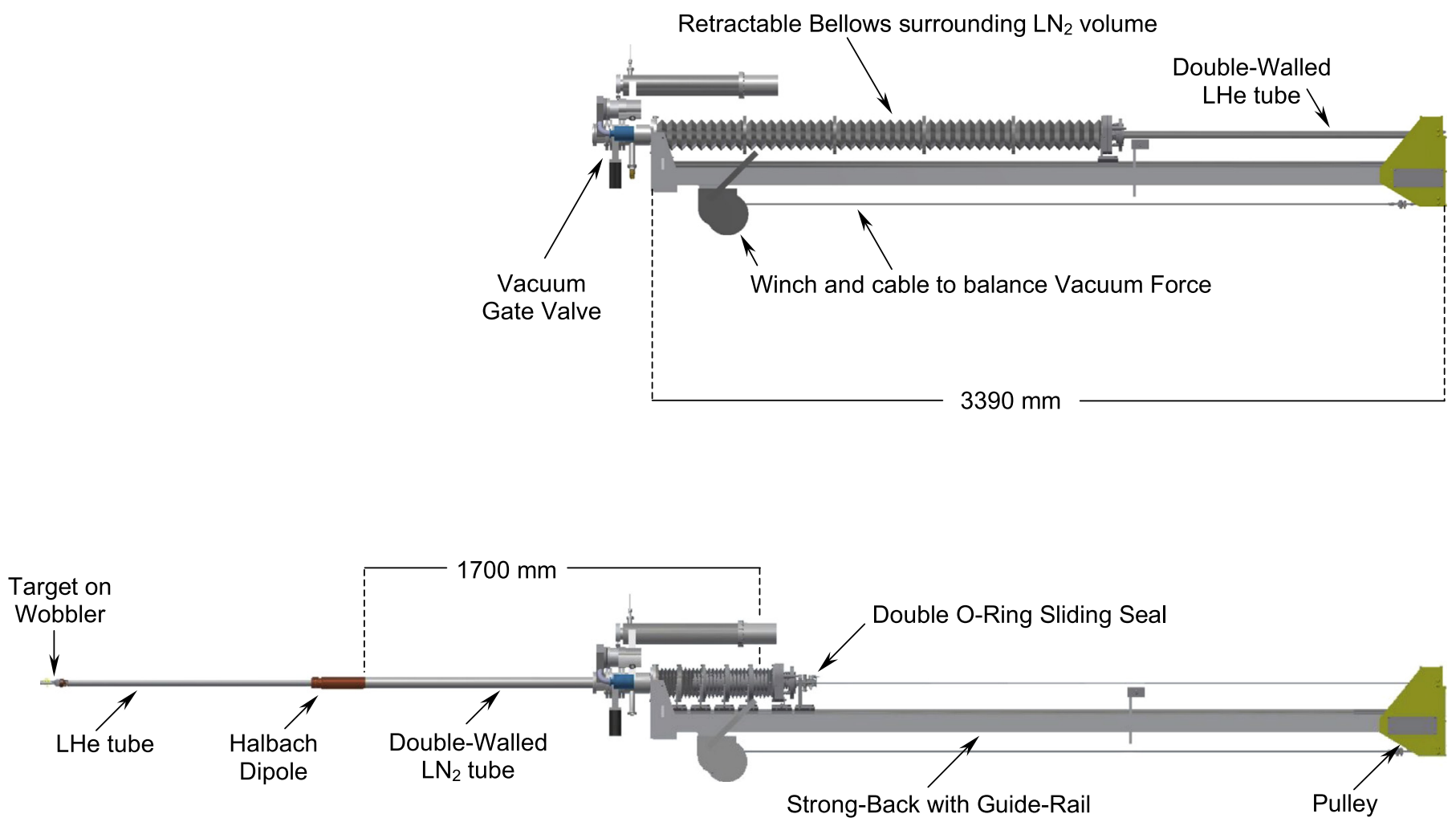

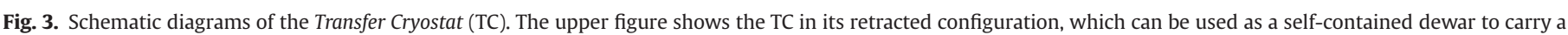

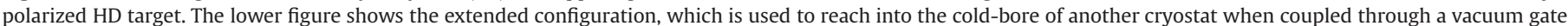
valve. During transfer operations, the TC is supported vertically with the top (containing the indicated Pulley) shown at the right of the figure. 
cryostats, a permanent magnet array in the TC provides a holding field for preserving target polarization.

The core design of the present TC was originally developed at BNL for handling frozen-spin HD targets during experiments at the Laser-Electron-Gamma-Source (LEGS) [5,9]. The overall dimensions of the TC were constrained by the geometry of the target cell, the dimensions of the cryostats used for production and polarization, and the requirement of maintaining target polarization during transport between cryostats.

Operational reliability is a crucial factor due to the number of successful transfers needed to place a polarized target in beam. As outlined in Section 2, the successful utilization of an HD target for an experiment usually requires five transfers with the TC. Because of the significantly shorter spin relaxation times under the TC conditions, the time elapsed in each TC transfer must be minimized. Earlier generations of TCs used with HD targets had transfer success rates between $65 \%$ and $75 \%$ [7-9]. Such efficiencies result in an overall success rate of less than $25 \%$ for five transfers, $(0.75)^{5}$, which makes the running of a major experiment difficult. Since its original construction and operation at BNL, several important additions and modifications have been incorporated into the design of the TC to improve operational reliability. The resulting device has a transfer success rate of about 95\%. Thus, five transfers using the current device succeed more than $75 \%$ of the time, which is an efficiency factor that can be accommodated within a major experiment. The current design described here was used during the E06-101 experiment [6] at JLab.

\subsection{Moveable cryogen volumes}

The liquid cryogen spaces of the TC are fabricated as a pair of concentric cylindrical and toroidal volumes surrounded by an insulating vacuum space formed from a series of highly compressible bellows, as shown in Fig. 3. The $3.3 \mathrm{~m}$ long central cylindrical volume forms a 1.1 liter LHe volume. The lower end of the LHe volume terminates in a mechanical connection to the target.

The top half of the LHe volume is jacketed by a second wall that is vacuum tight at the upper end but is open to vacuum at the lower end. A port at the top of the volume allows the insertion of a liquid helium transfer lance for cryogen filling. During TC cooling, the transfer lance mates to a $1.3 \mathrm{~m}$ long permanent thin-walled tube that effectively extends the lance and directs the LHe to the bottom of the volume. This prevents vapor blockages and enables a rapid cool-down. Additional ports at the top of the volume contain an electrical feedthrough for temperature and liquid-level sensor leads and a vent for helium boil-off.

Surrounding the LHe volume and separated by vacuum is a $320 \mathrm{~mm}$ long, 1.81 toroidal liquid nitrogen $\left(\mathrm{LN}_{2}\right)$ volume that is mechanically attached to the top of the bellows assembly. A $1380 \mathrm{~mm}$ long, double-walled tube extends down from the bottom of this toroidal volume providing a $77 \mathrm{~K}$-shield with a total length of $1700 \mathrm{~mm}$ for the $\mathrm{LN}_{2}$ volume. It is this extended section that enters a mating cryostat during target insertion or extraction operations. Two $3 \mathrm{~mm}$ thick insulating spacers made of G10 epoxy fiberglass are mounted on the inside surface of the $\mathrm{LN}_{2}$ extension. The spacers are separated by $50 \mathrm{~cm}$ and align the central LHe tube by forming a slightly larger aperture at four points on the circumference. A set of three access tubes extends from the $\mathrm{LN}_{2}$ volume through the top of the vacuum bellows assembly. Two of the tubes serve as fill and vent lines for liquid nitrogen, and the third tube carries the leads for a capacitive liquid level sensor for the toroidal volume.

The LHe and $\mathrm{LN}_{2}$ volumes are insulated from room temperature by a $15 \mathrm{l}$ vacuum space formed by a $1.9 \mathrm{~m}$ long series of highly compressible welded bellows, a sliding seal assembly at the top of the bellows, and a gate valve at the bottom of the TC. This surrounds the jacketed length of the LHe volume. A winch at the bottom of the TC supports the vacuum bellows assembly against vacuum forces through a cable via a pulley located at the top of the TC. This winch and cable system controls the motion of the LHe volume and the bellows. Compression of the bellows permits vertical motion of the top of the bellows assembly, the connected $\mathrm{LN}_{2}$ volume, and the central LHe volume, which penetrates through the sliding seal assembly at the top of the bellows. The configurations of the TC in its fully retracted and fully extended positions are shown in Fig. 3. In the latter configuration, the TC can extend a $2 \mathrm{~K}$ connection deep into the cold regions of all cryostats used with polarized HD. During TC extraction, the mechanical advantage of the winch is used to pull the LHe tube up against the vacuum force. When a stainless steel ring, clamped around the outside of the LHe volume at a point $2 \mathrm{~m}$ above the target, meets the top plate of the bellows assembly, the LHe volume is then entirely within the $\mathrm{LN}_{2}$ volume. Further action of the winch then expands the bellows as it raises both $\mathrm{LHe}$ and $\mathrm{LN}_{2}$ volumes.

The weight and motions of the TC are supported and guided by a $100 \mathrm{~mm} \times 50 \mathrm{~mm}$ by $3.3 \mathrm{~m}$ long aluminum strong-back tube that spans the length of the cryostat. On top of the strong-back is a linear track. Five sliding bearing blocks ride the track and guide and support the vacuum bellows assembly. Additionally, the top of the LHe volume is guided and supported by a sliding bearing block riding on the track.

The outer jacket wall of the LHe volume makes contact with a pair of sliding O-ring seals at the top of the bellows assembly. This set of seals allows translation and rotation of the LHe volume and the target attachment screw thread at its lower end. A pump-out port is located between the pair of sliding seals and creates an evacuated region that is separated from the main vacuum space.

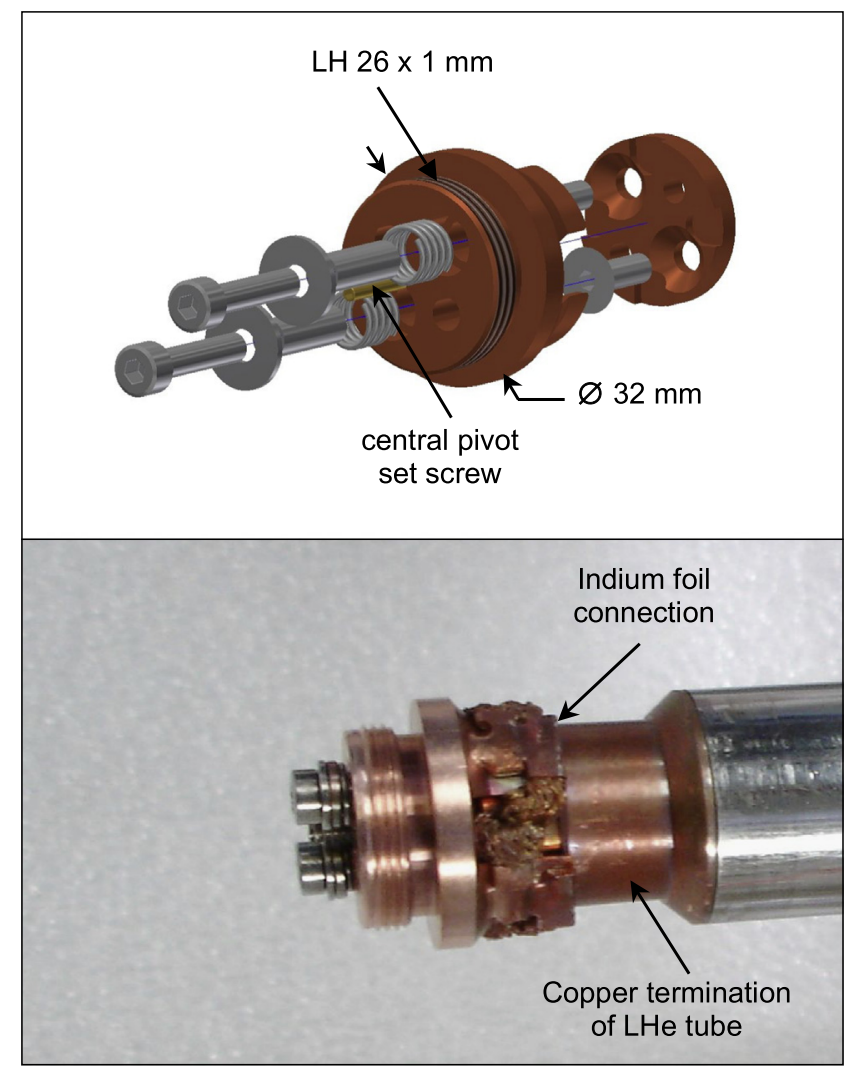

Fig. 4. An exploded-view and photo of the low-temperature Wobbler connection A copper disk with left-handed threads pivots against a copper disk that is connected to the bottom end of the LHe volume with an indium joint. The two disks are thermally coupled by a set of four copper braids (visible in the photo) that are electron-beam welded to each disk. 
The vacuum space can be evacuated through a gate valve located at the bottom of the TC using an onboard turbo-molecular pump (Agilent/Varian M81). The vacuum space is connected to the interior of the strong-back tube, which adds 161 of expansion volume. A relief valve prevents over-pressurization in the event that the target is vaporized due to loss of cooling.

\subsection{TC connection to the target - the Wobbler}

Engaging the inner $\mathrm{LH}$ threads of a target cell with the matching threads on the end of the LHe volume is accomplished by rotating a section of the vacuum-jacketed LHe tube that is exposed above the TC bellows assembly. A flexible connection or Wobbler is mounted on the bottom of the LHe volume. The Wobbler (Fig. 4) is made from two copper disks whose relative angular alignment is maintained by a pair of axial screws. A co-axial set-screw provides a $1 \mathrm{~mm}$ stand-off between the disks and forms a central pivot point. Springs mounted on each of the axial screws provide tension between the disks. The lower disk has a set of left-handed M26 $\times 1$ threads that match the internal threads of a target cell. The two disks are thermally connected by four copper braids that have been electron-beam welded to both disks. The Wobbler assembly is bolted to a copper termination on the end of the LHe volume, and a $50 \mu \mathrm{m}$ thick indium foil ensures good thermal contact.

The Wobbler compensates for slight misalignments between the TC and a cryostat during target transfer operations and prevents cross-threading. The pivoting action allows the TC to "find" the correct starting point within a target thread. In addition, the target threads are also machined with a blunt start to help define the proper engagement. During the tightening of the Wobbler connection to the LHe volume, the joint is nominally heated to approximately $140{ }^{\circ} \mathrm{C}$, slightly below the indium melting point of $156^{\circ} \mathrm{C}$, to ensure that the indium can flow and fill the gap between surfaces. Because the critical field of indium [11] is well
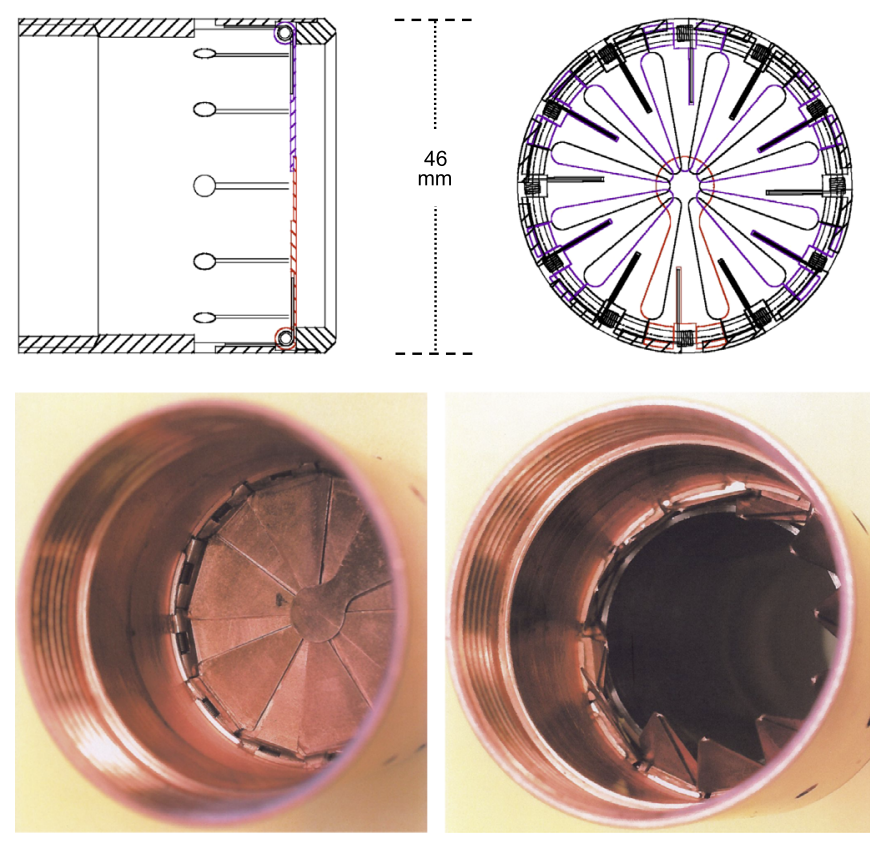

Fig. 5. Spring-loaded inward-opening $77 \mathrm{~K}$ radiation shutter; side view (top-left) and an end view, looking in from the back (top-right). The shutter has eleven identical overlapping leaves and a longer twelfth one that covers the central gap. A photo of the shutter viewed from the back (the target side) in its closed position is shown in the lower-left panel. The lower right panel shows the shutter with its 12 spring-loaded leaves forced partially inward when pressed against a shutteropener. below the fields in the TC, the indium does not become superconducting, which would generate a thermal break.

\subsection{K-Shield}

Threaded into the base of the $\mathrm{LN}_{2}$ volume is a cylindrical rareearth permanent dipole magnet, which is discussed in Section 3.5. Connected to the bottom of the magnet cylinder is a circular springloaded shutter fashioned from 12 overlapping leaves, as shown in Fig. 5. The shutter leaves can be rotated inward against the spring action; when pressed against a thin ring (a shutter-opener) during target transfer operations, the shutter is mechanically opened. Shutter-openers are installed within each cryostat at locations that are $40 \mathrm{~K}$ or colder. This design is similar to an 11-leaf assembly described in [7]. When closed, the shutter functions as an extension of the $77 \mathrm{~K}$-shield and prevents $300 \mathrm{~K}$ thermal radiation from being incident on a target held within the TC.

Operational experience has revealed that about $1 / 3$ of the time, one or more of the spring-loaded leaves fail to completely close when the cooled shutter is lifted off a shutter-opener ring within a cryostat, which could expose the target to $300 \mathrm{~K}$ radiation. An auxiliary $\mathrm{LN}_{2}$ baffle has been incorporated above the TC gate valve (Fig. 6) in order to minimize the impact of shutter closure failures. This auxiliary $\mathrm{LN}_{2}$ baffle is formed from a cylindrical brass tube ending in a sliding copper plate which are both conductively cooled by contact with an external $\mathrm{LN}_{2}$ volume mounted on the side of the TC. Once the TC has been completely retracted (see Fig. 3), the auxiliary $\mathrm{LN}_{2}$ baffle plate is pushed into position, sealing off the bottom of the $\mathrm{LN}_{2}$-cooled brass tube and effectively capturing the shutter within a $\mathrm{LN}_{2}$-cooled cup. In this way, the exposure to $300 \mathrm{~K}$ radiation from any gaps between shutter leaves is limited in duration to about $30 \mathrm{~s}$, which is the time needed to raise the shutter off from its cold "opener"-ring and fully retract it behind the auxiliary $\mathrm{LN}_{2}$ baffle.

\subsection{Vacuum lock connection}

A full view of the lower section of the TC is shown in Fig. 7, with a cut-away model on the left and a photo of the assembled unit on the right. The bottom ISO-63 flange attaches to a matching flange on a receiver cryostat. During the cycle of an HD target, the polarization relaxation times are the shortest during the periods spend in the low-field and comparatively high temperatures of the TC. Minimizing the elapsed time in the TC reduces the potential polarization loss during target transfers. For this reason it is important

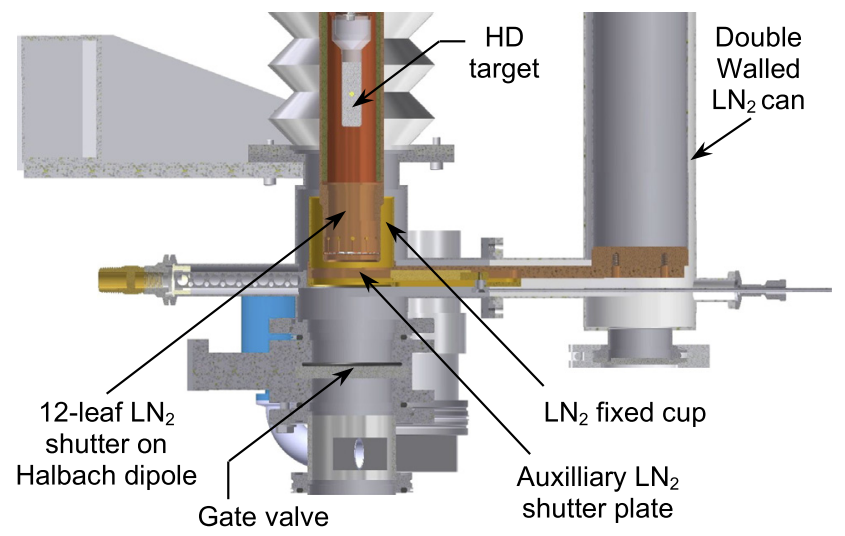

Fig. 6. Full $77 \mathrm{~K}$ shield. In the retracted position, the target is centered in the $77 \mathrm{~K}$ dipole magnet. The leaf shutter of Fig. 5 is threaded onto the dipole, which is directly connected to the TC tube containing $\mathrm{LN}_{2}$. The shutter is captured in an auxiliary cup, which is conduction cooled from a separate $\mathrm{LN}_{2}$ volume. 


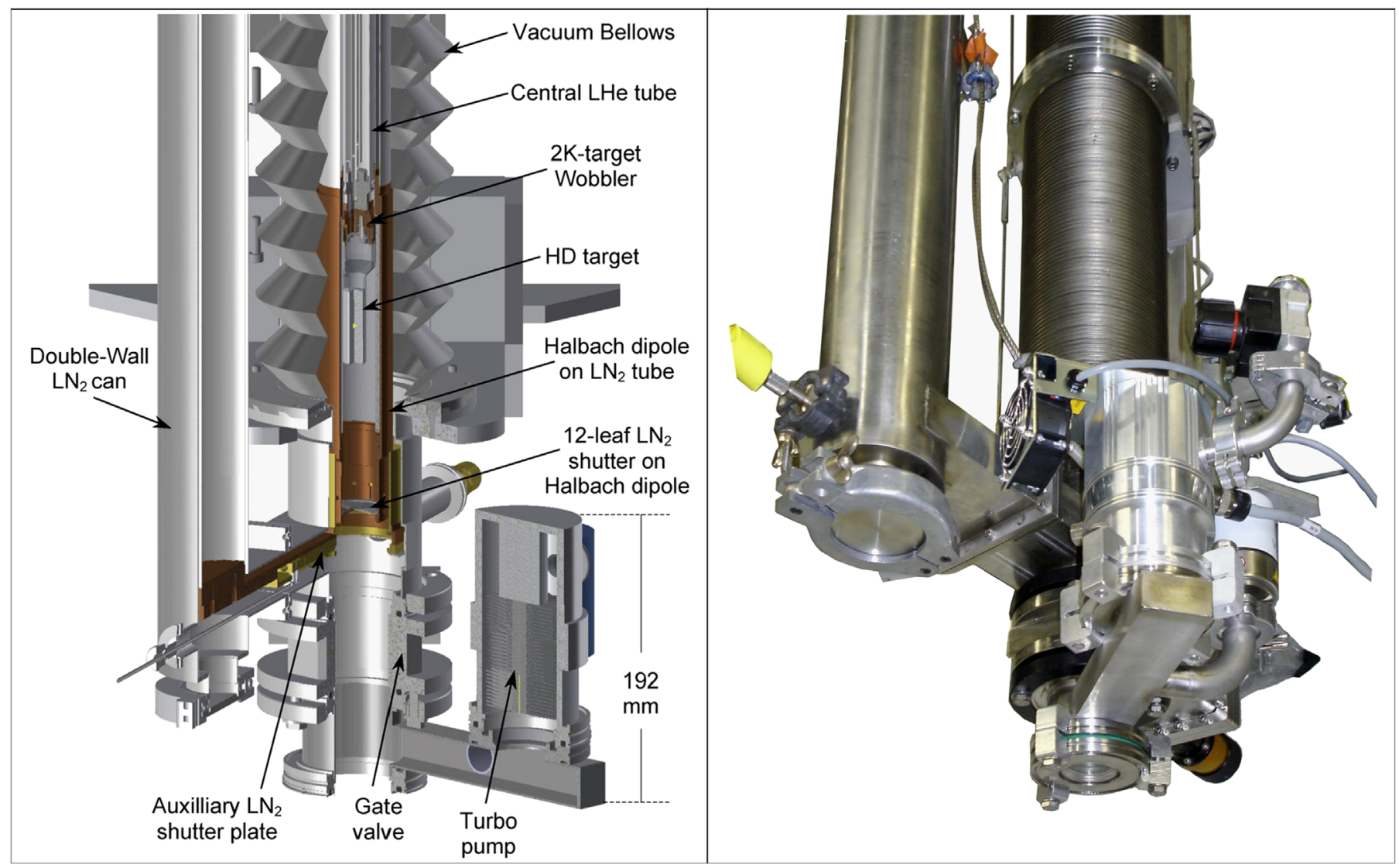

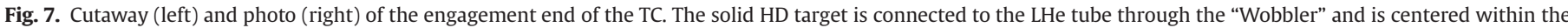

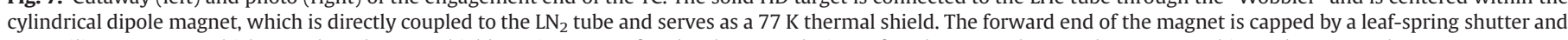

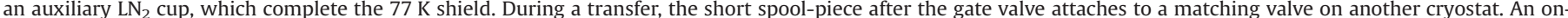

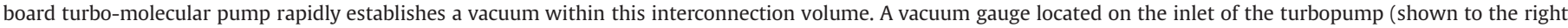
and behind the turbopump) provides a monitor of the pressure from atmosphere into the $10^{-7}$ mbar range.

to minimize the time devoted to simply re-establishing a good vacuum in the connection between the TC and its mating cryostat. For rapid pump down, an on-board turbo-molecular pump (Agilent/ Varian 81-M) is directly mounted on the short spool piece connecting the TC gate valve and the matching gate valve on a receiver dewar. This brings the interlock pressure into the $10^{-6}$ mbar range within a few minutes, as measured by a cold-cathode gage mounted directly on the spool piece extension.

\subsection{Halbach magnet}

For frozen-spin HD, magnetic fields greater than about 100 Gauss are needed to prevent rapid HD polarization loss. Within the TC and attached to the bottom of the $\mathrm{LN}_{2}$ heat shield is a cylindrical permanent magnet array, arranged in one of the Halbach configurations designed to produce an internal dipole field [12] of approximately $0.1 \mathrm{~T}$. The array consists of 16 sectors of neodymium magnets that are mounted in a copper shell. The magnetization direction for each of the neodymium alloy sectors is shown schematically in Fig. 8. Relative to the top sector, each other sector located at an azimuthal angle $\phi$ has a field direction that is rotated by $2 \phi$. This produces a net dipole field in the interior of the cylinder that is transverse to the axis.

The magnetic material is an alloy of neodymium, iron, and boron (NdFeB), obtained from Tridelta Magnetsysteme [13]. A roomtemperature solenoid, top panel of Fig. 9, has been used to magnetize the alloy. Four bars of the NdFeB alloy are held in the desired orientation transverse to a plastic sleeve, shown here partly inserted into the bore of the air-core solenoid. A current pulse from the

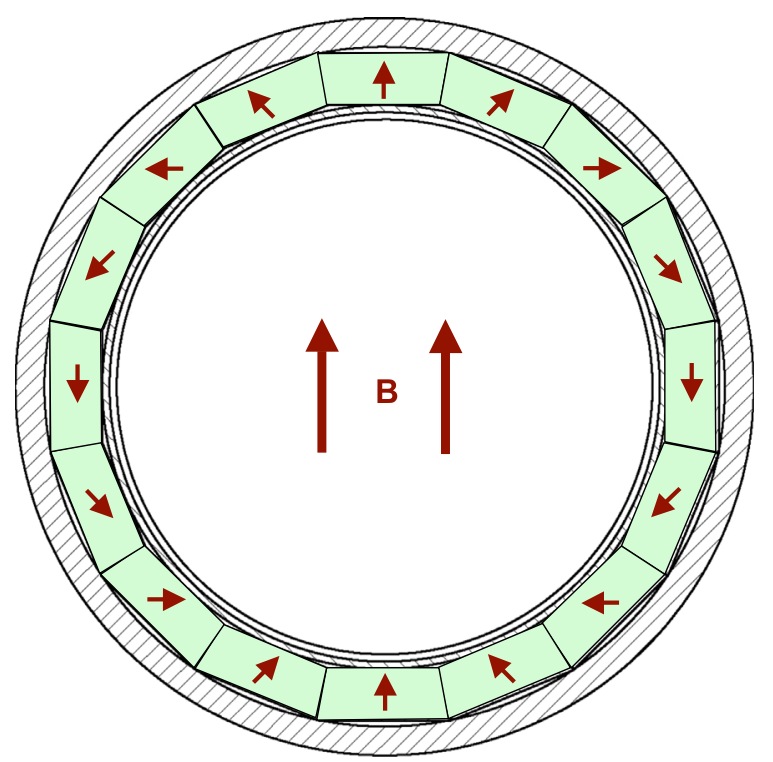

Fig. 8. Cross-section of the TC magnet made from 16 sectors of neodymium magnets. The directions of magnetization in each sector are indicated by the arrows. This Halbach configuration produces a net transverse dipole field within the array.

discharge of a capacitor [14] generates the field pulse shown in the middle panel of Fig. 9. The $3.3 \mathrm{~T}$ peak field exceeds the saturation level of the material and results in a permanent 


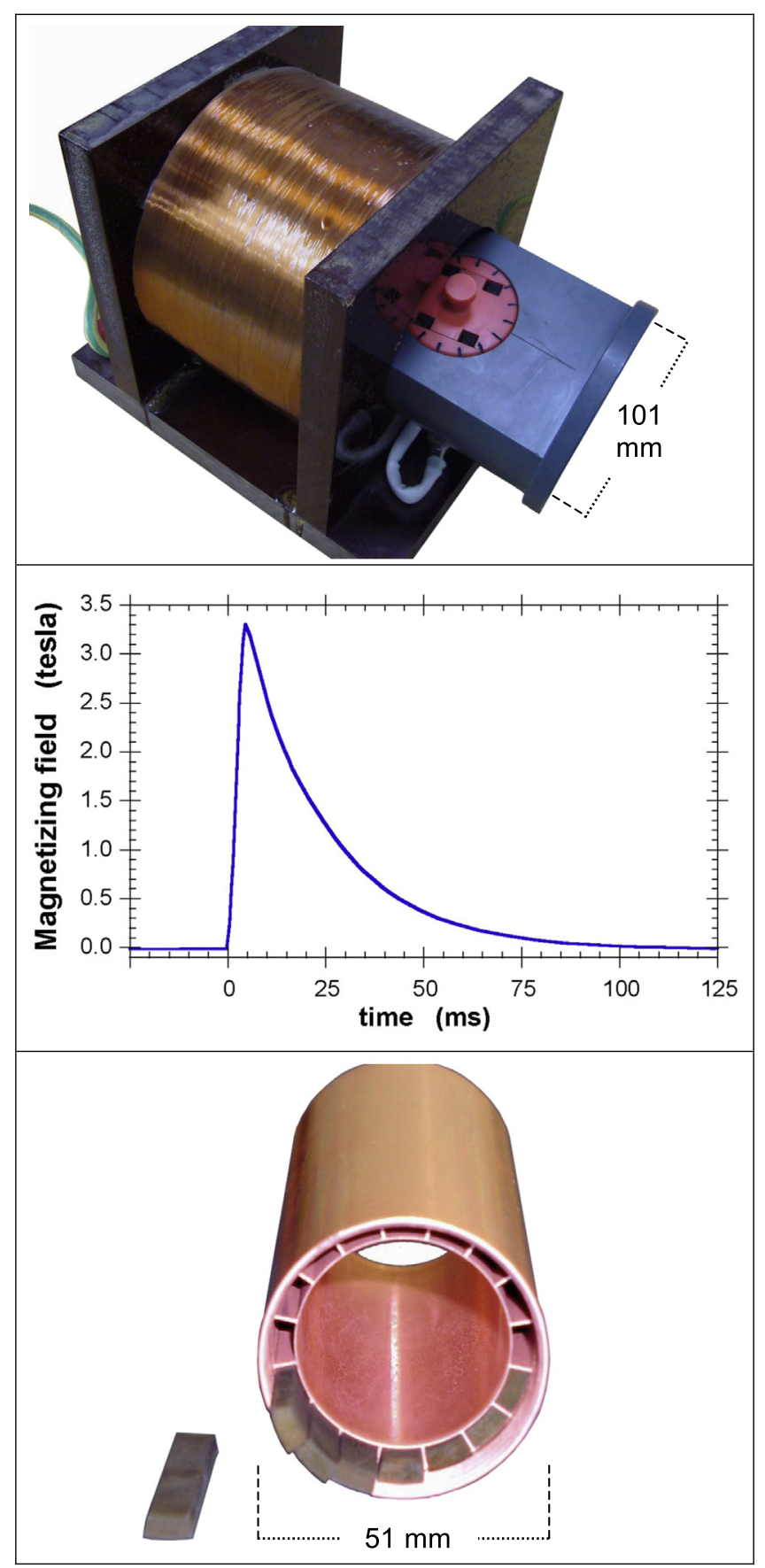

Fig. 9. The solenoid used to magnetize sections of neodymium alloy (top panel), shown with a fixture that holds up to four sections in the desired orientation. A typical magnetizing pulse is plotted in the center panel. The magnetized sections are captured within a copper sleeve (bottom panel) to create a Halbach dipole with a field transverse to the axis of the assembly - see text.

magnetization. The neodymium alloy bars are machined with a trapezoidal cross-section and, once magnetized, are captured within channels that have been wire-cut from a thick-walled copper tube, as shown in the lower panel of Fig. 9. Each of the 16 channels contain three bars end to end that run the length of the magnet assembly.

The room-temperature fields generated by the Halbach array are plotted in Fig. 10. On axis, the field is flat over the central $80 \mathrm{~mm}$ length at about $1.1 \mathrm{kG}$. When the TC is fully retracted, targets are centered near the $z=80 \mathrm{~mm}$ position. HD target diameters used at BNL and JLab have varied in size with radii between $7.5 \mathrm{~mm}$ and $12.5 \mathrm{~mm}$. The field at an average radius of
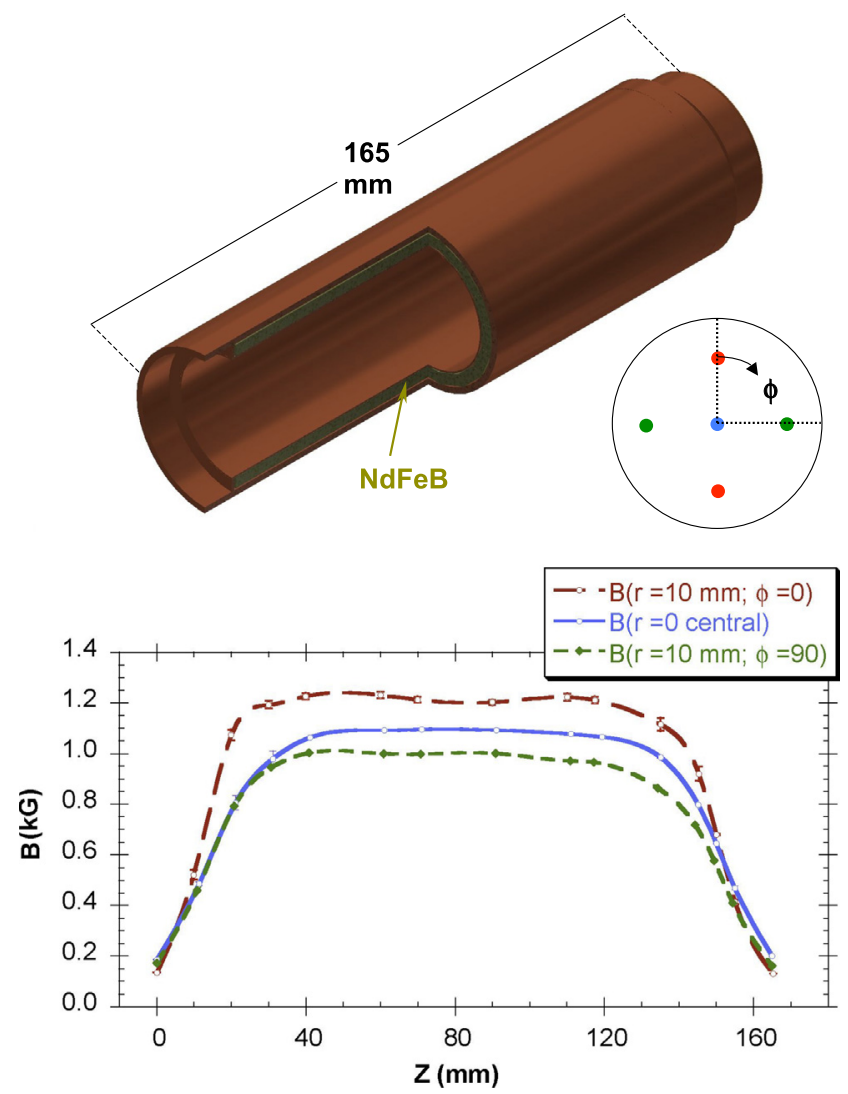

Fig. 10. Cutaway schematic of the Halbach dipole and its internal room-temperature fields. In the retracted position, a solid HD target would be centered at about $z=80 \mathrm{~mm}$.

$10 \mathrm{~mm}$ from the central axis varies by about $10 \%$ due to variations in magnetization of the neodymium sectors.

The fields produced by neodymium magnets are temperature dependent and have a maximum field strength at about $135 \mathrm{~K}$, where the alloy undergoes a spin-reorientation [15]. This phase transition is reversible as the magnet is thermally cycled. Measurements of the magnets used in this Halbach dipole are summarized in Fig. 11. At temperatures below the phase transition, the field strength decreases monotonically. At $4 \mathrm{~K}$, the field strength of the magnets is approximately $90 \%$ of the value at $300 \mathrm{~K}$; at $77 \mathrm{~K}$ (the nominal temperature of the Halbach dipole in

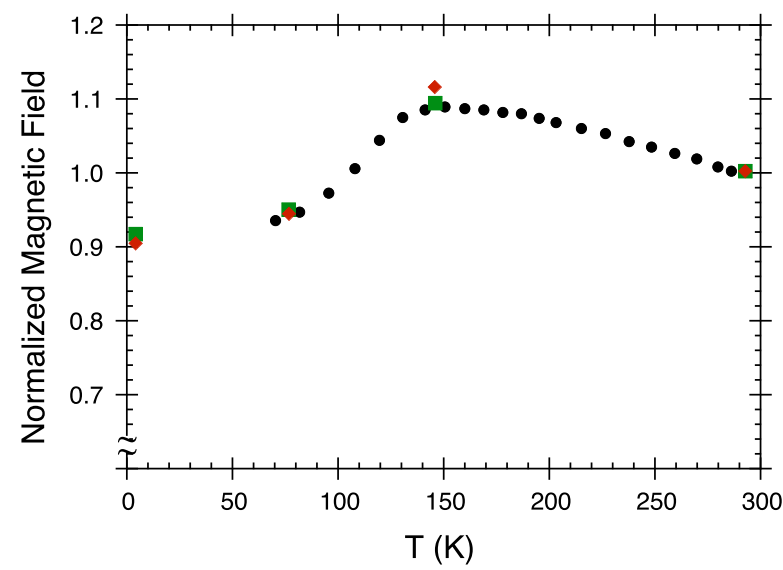

Fig. 11. The temperature dependence of the field strength for neodymium magnets. Different magnets were made from samples of neodymium alloy as measured with different Hall-probes. Black circles were measured with a probe in a flowing bath of room temperature gas. Data shown as squares and diamonds were measured with a temperature-compensated cryogenic Hall probe. 
the TC during transfer operations), the field strength is approximately $95 \%$ of that at $300 \mathrm{~K}$.

The TC extracts polarized HD targets from cryostats with longitudinal magnetic fields. Because the motion of the target cell during extraction is negligible compared to the Larmor frequencies for $\mathrm{H}$ and $\mathrm{D}$, the spins adiabatically rotate as they enter the TC Halbach transverse dipole. Thus, the Halbach dipole brings two important characteristics to the TC: it provides an adequate holding field in a limited radial space, and its interior transverse orientation avoids zeros in the magnetic field that could occur between two solenoids with unintentionally opposed directions.

\subsection{Counterbalanced lifting arm}

During transfer operations a retractable arm is used to position the TC off-center from a hoist to eliminate mechanical interference from crane components (see Fig. 12). The lifting point slides on rails and is positioned using a motor and lead screw. Lead counterweights are fixed on the free end of the lifting arm. When the TC is supported by a hoist and the lifting arm is extended, adjustments in the position of the lifting point on the arm produce vertical movements of the TC. The large mechanical advantage provides a delicate control when docking the TC on the top of another cryostat.

For service operations, the lower end of the TC is connected to the free end of the lifting arm by an approximately $1 \mathrm{~m}$ long stainless-steel cable. With the cable in place, movement of the lifting point along the arm causes the TC to tilt. In this way the TC

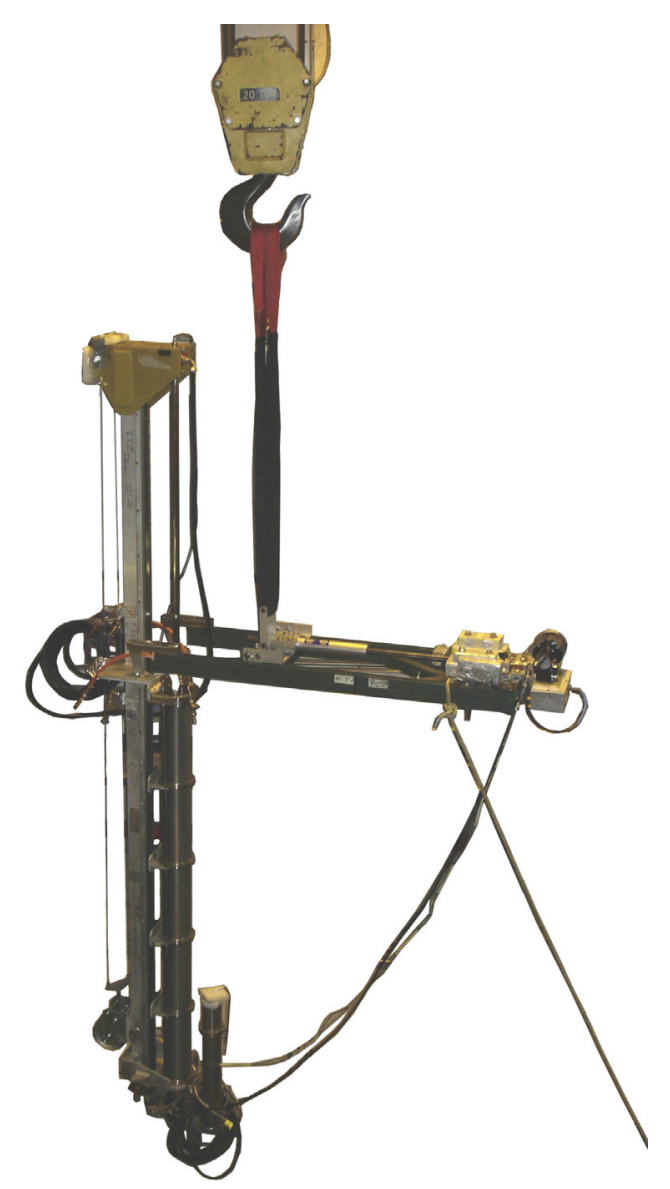

Fig. 12. Photo of the counter-balanced lifting arm used to position the Transfer Cryostat off-center from a hoist. The adjustable lifting point with its counter weight provides the delicate control needed to connect the TC to another cryostat. can be maneuvered to a $30^{\circ}$ position for service, cooling, or storage on a cart. Movement of the lifting point to its farthest position on the arm allows a horizontal orientation of the TC, which permits a rapid removal of cryogens, if required.

\subsection{Instrumentation}

The cryogen levels in the volumes of the TC are continuously monitored during operations. A capacitance level meter [16] measures the $\mathrm{LN}_{2}$ level, and a superconducting wire [16] measures the LHe level. The temperature of the LHe tube is monitored with a Cernox resistor [17]. During transfer operations, the partial pressures of mass-3 (HD), mass- $4\left({ }^{4} \mathrm{He}\right)$, mass-28 $\left(\mathrm{N}_{2}\right)$ and mass-32 $\left(\mathrm{O}_{2}\right)$ are continuously monitored with a residual gas analyzer (Stanford Research Systems RGA-100 [18]) that samples the outlet of the turbopump that is pumping the space containing the target (Fig. 7).

\section{Operation and performance}

\subsection{Preparations and cooling}

After each thermal cycle of the TC, the Wobbler connection to the LHe tube is tightened to $3.4 \mathrm{~N} \mathrm{~m}$ at $140{ }^{\circ} \mathrm{C}$, and then again at room temperature to ensure good contact across the indium interface as discussed in Section 3.2. Then, with the spool piece at the bottom of the TC blanked off and the vacuum-gate valve open (Fig. 7), the central space is evacuated using the on-board turbopump to less than $5 \times 10^{-7}$ mbar. The cable and winch mechanism indicated in Fig. 3 holds the moveable volumes in place against the vacuum force.

The $\mathrm{LN}_{2}$ and LHe volumes are next purged of condensed water from previous use by repeated cycles of pumping to $0.05 \mathrm{mbar}$ and backfilling with $1 \mathrm{~atm}$ of helium gas. TC cooling takes place with the TC supported on a cart at an angle of $30^{\circ}$ from horizontal. The liquid nitrogen volume is first filled with $\mathrm{LN}_{2}$. The LHe volume is next pre-cooled with cold helium gas and then filled with LHe. The average time to cool to $4 \mathrm{~K}$ is approximately $45 \mathrm{~min}$.

Once the TC has reached a stable temperature of $4 \mathrm{~K}$, the auxiliary $\mathrm{LN}_{2}$ volume is filled. Just prior to the start of a transfer operation, a roughing pump is connected to the back of the central LHe tube and the TC temperature is lowered to $2 \mathrm{~K}$, which takes about 20 min to stabilize. The TC can then be lifted from its cart with the fixture discussed in Section 3.6. Once in a vertical position, the TC is ready to be positioned over a cryostat for a target transfer operation.

\subsection{Cryogenic hold times}

The internal TC $\mathrm{LN}_{2}$ volume has an average hold time of $1.3 \mathrm{~h}$ and is typically topped off once during a transfer. The auxiliary $\mathrm{LN}_{2}$ volume will remain cold for over $6 \mathrm{~h}$. With the $\mathrm{LN}_{2}$ volumes cold, the LHe volume has a hold time of about $18 \mathrm{~h}$ at $4 \mathrm{~K}$. When the central LHe volume is pumped to lower the temperature to $2 \mathrm{~K}$ there is an initial $40 \%$ reduction in the LHe level. From that point on, the $2 \mathrm{~K} \mathrm{LHe}$ hold time is about $10 \mathrm{~h}$. These times are sufficient for the transfer of a solid polarized target between two cryostats, which takes about $2 \mathrm{~h}$.

\subsection{TC insertion into a cryostat}

Once the spool piece under the TC gate valve (Fig. 7) is mated to a matching vacuum gate valve on another cryostat, the space between the two gate valves is evacuated, by first roughing through the onboard turbopump and then to high vacuum with the turbopump spinning. The gas exiting the turbopump is monitored with an RGA (Section 3.7). Three stabilizing chains anchored to fixed points near 
the cryostat are attached to the top section of the TC and their tensions adjusted to level the TC so that its axis is held rigidly parallel to that of the cryostat. Aligning and fixing the TC position is essential in order to constrain the motion of the TC LHe tube when extended, as in Fig. 3. (A poor alignment of the TC with the mating cryostat can lead to excessive friction when rotating the target threads. This can generate thermal spikes, which can raise the HD into the temperature region where the polarization can decay more rapidly due to a shortened $\mathrm{T}_{1}$ or even material can be lost from the target cell due to high vapor pressure. This is partly mitigated by operating at $2 \mathrm{~K}$, as discussed in the next section.)

Once the pressure between the gate valves has been reduced below $5 \times 10^{-6}$ mbar (within a few minutes, or in practice by the time the stabilizing chains have been adjusted) the lower gate valve of the connecting cryostat is first opened and the residual gas is sampled to verify a similar or lower pressure, particularly for He. (Variable temperature cryostats such as the PD, discussed in Section 2.1, are typically operated with a few mbar of He that provides a thermal exchange between the HD and a separate LHe reservoir. This exchange gas must be thoroughly pumped away before beginning transfer operations, since any residual He entering the TC during the transfer process could thermally short its LHe tube and result in a subsequent loss of cryogens. Depending upon conditions, this preparatory exchange gas pumping can take many hours since the vapor pressure of cold He is quite low.) The TC gate valve is then opened and the shutter plate of the auxiliary $\mathrm{LN}_{2}$ shield is retracted. The TC bellows assembly is then allowed to compress under the vacuum force by unspooling the support cable using the winch shown in Fig. 3. As it does so, the $\mathrm{LN}_{2}$ and $\mathrm{LHe}$ volumes extend into the cryostat. This continues until the shutter (Fig. 5) seats in the shutter-opener ring of the cryostat, which mechanically opens the 12 spring-loaded leaves (Fig. 5). The shutter-opener now supports the TC $\mathrm{LN}_{2}$ volume and no further compression of the TC bellows is possible. Each HDice cryostat has a shutter-opener ring anchored to a point with a temperature of $40 \mathrm{~K}$ or lower, and coils that guarantee a continuous coverage of magnetic field below the TC Halbach dipole.

Further extension into the connecting cryostat is accomplished by sliding the LHe tube down through the double O-ring seal. During this procedure, the space between the two O-rings is evacuated and the pressure at the inlet to the roughing pump is continuously monitored. A counter-weight is attached to the cable above the LHe tube to offset the vacuum force and provide better control. The LHe tube is lowered until the Wobbler (Fig. 4) contacts the target. The LHe tube is then rotated clockwise (against the LH threads) until the starting thread on the Wobbler falls into the blunt start of the LH target thread. Subsequent counter-clockwise rotation of the LHe tube engages the Wobbler threads in the inner threads of the target ring (Fig. 1).

\subsection{Thermal spikes from thread engagement at $2 \mathrm{~K}$}

The temperature of the connecting cryostat is continuously monitored at a point close to the target threads. Turning the threads of the Wobbler against the copper target ring generates heat. It is important that this motion be smooth and moderately slow to allow the heat to dissipate. When the TC is used at $4 \mathrm{~K}$ (without LHe pumping), it is difficult to avoid spikes in target temperature that reach into the $6-7 \mathrm{~K}$ region where the vapor pressure of $\mathrm{HD}$ becomes appreciable $\left(10^{-3} \mathrm{mbar}\right.$ at $\left.7 \mathrm{~K}\right)$. In contrast, when the TC LHe volume is pumped to reach $2 \mathrm{~K}$, the added cooling from the TC keeps the thermal spikes generated by inter-thread friction below $4 \mathrm{~K}$ where the $\mathrm{HD}$ vapor pressure is negligible $\left(5 \times 10^{-9} \mathrm{mbar}\right)$. With repeated counter-clockwise rotation of the TC LHe tube, the left-hand (LH) Wobbler threads bottom in the inner LH threads of a target ring (Fig. 1). Additional torque is then needed to break the right-hand ( $\mathrm{RH}$ ) target threads free from the cold pedestal of the connecting cryostat, which results in another temperature spike. As counter-clockwise rotation of the TC LHe tube continues, the target threads out of the cryostat pedestal. The associated temperature spikes tend to be larger at this stage because the larger radius RH target threads have a greater contact surface area. Once the target is completely disengaged from the cryostat pedestal, its temperature is then entirely determined by the $2 \mathrm{~K}$ TC LHe tube. It is then pulled up, first into the TC Halbach dipole by raising the LHe tube with the TC winch system. (In this process, the outside of the TC LHe tube that has been cooled by exposure to the environment of the connecting cryostat has to be pulled through the TC double O-ring sliding seal, indicated at the top of the bellows assembly in Fig. 3. To prevent the O-rings from freezing, heated air is used to warm the exterior region of the sliding seal prior to and during extraction of the TC LHe tube. The rate of extraction must be adjusted by monitoring the pressure in the line pumping the space between the O-rings of the sliding seal.) Continued use of the winch raises the $\mathrm{LN}_{2}$ volume, allowing to spring close the 12-leaf shutter and bringing the target completely up into the TC, after which the auxiliary $\mathrm{LN}_{2}$ shutter plate is closed to complete the $77 \mathrm{~K}$ thermal shield around the target. Finally, the vacuum gate valves on the TC and the cryostat are closed.

At this point the TC with its polarized target can be moved to another cryostat. Inserting the target into another receiver cryostat follows the same sequence as outlined above, except that the TC LHe tube is rotated clockwise to deposit the target and disengage the TC Wobbler.

\subsection{Performance with frozen-spin $H D$}

Of the approximately $2 \mathrm{~h}$ transfer process, the average time during which the temperature and magnetic field seen by an HD target is determined by the TC is about $50 \mathrm{~min}$. To study the polarization loss due to spin-lattice relaxation $\left(T_{1}\right)$ while the target is in the TC, several test lifts were performed with frozen-spin HD targets. Typical results are shown in Fig. 13, with $\mathrm{H}-$ and Dpolarizations monitored by field-sweep NMR. The area of the resulting NMR signal for $\mathrm{H}$ is proportional to the $\mathrm{H}$-polarization and is plotted in the upper panel, and the corresponding Dpolarization signal is shown in the lower panel. The NMR data collection required a few minutes and in between these periods the target was held in a cryostat at $2 \mathrm{~T}$ and $3 \mathrm{~K}$.

After approximately 1 day (the $25.3 \mathrm{~h}$ point in Fig. 13), the target was extracted from the cryostat, lifted up into the TC and held there for $50 \mathrm{~min}$ before being returned to the cryostat for further NMR measurements. The data points shown as open circles in the figure were collected before this test lift. The curves were fitted to the pre-lift data, parameterized as $P(t)=P_{o} e^{-t / T_{1}}$ and extrapolated out into the post-lift period. While the spinrelaxation times were very long, and as a result could only be roughly determined in a day $(327 \pm 34 \mathrm{~h}$ for $\mathrm{H}$ and $2035 \pm 1283 \mathrm{~h}$ for $D$ ), it was clear that the polarization signals measured after the test lift (the solid diamonds in Fig. 13) were completely consistent with the expected dependence. The fractional polarization losses for both $\mathrm{H}(-1 \pm 2 \%$, relative $)$ and $\mathrm{D}(0 \pm 3 \%$, relative) during the transfer operation were insignificant.

The transfer operations with the TC are the most delicate stages in the HD target cycle and require the monitoring of many separate parameters. As such, depending upon the proximity of the source and the receiver cryostats, a reliable transfer typically requires between 5 people, when the cryostats are next to each other as shown schematically in Fig. 2, and up to 9 people when loading a target into a large experimental hall during which separate operations must be performed at multiple elevations. 

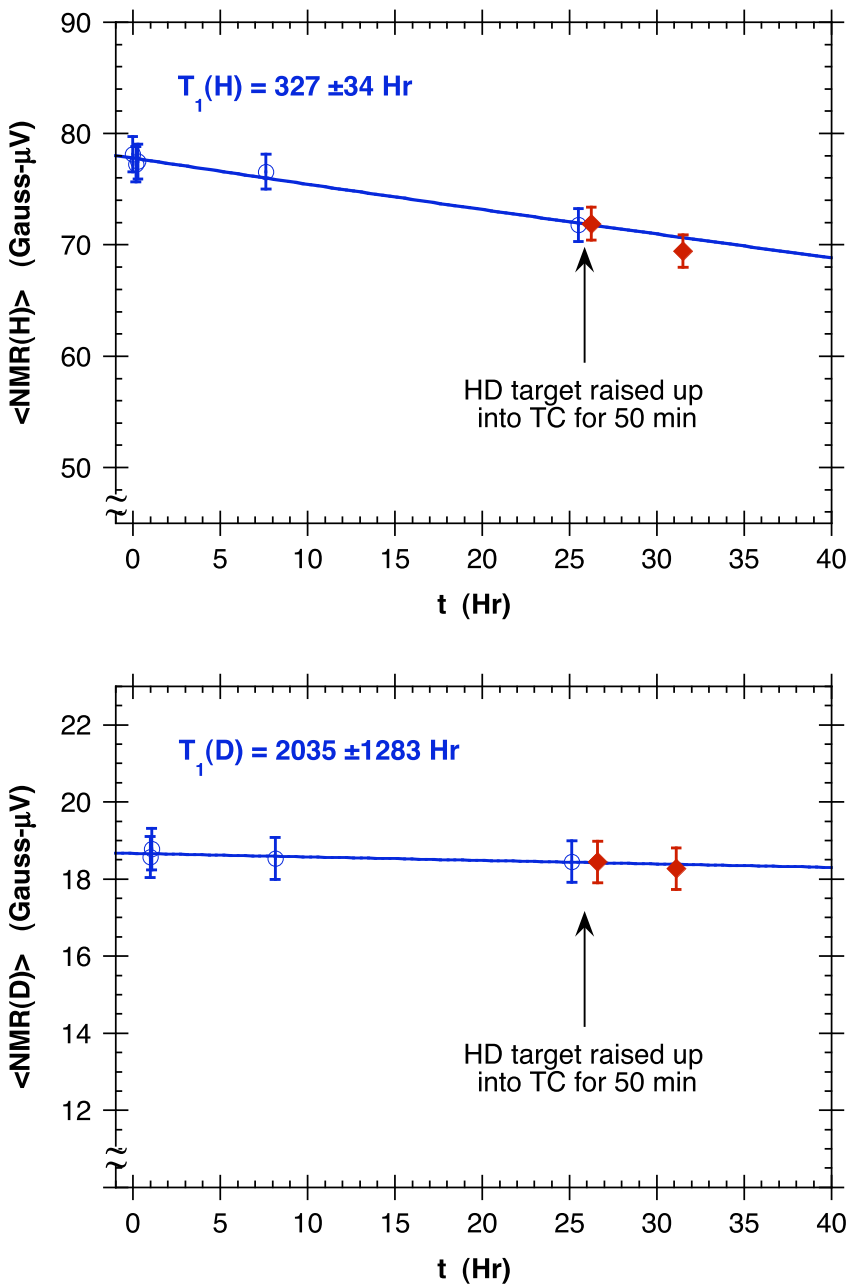

Fig. 13. Time dependence of the $\mathrm{H}$ (top panel) and $\mathrm{D}$ (bottom panel) polarizations of a frozen-spin HD target in a cryostat at $3 \mathrm{~K}$ and $2 \mathrm{~T}$. At the $25.3 \mathrm{~h}$ point, the target was extracted from the cryostat, held in the TC for $50 \mathrm{~min}$ and then returned to the cryostat. The data shown as open circles represent the areas of NMR signals before this test lift and the solid lines were fits to the expected relaxation, $P(t)=P_{0} e^{-t / T_{1}}$, extrapolated here into the post lift period. The solid diamonds were measured after the target was returned to the cryostat.

The potentially vulnerable steps in the transfer process have been discussed above, along with our mitigating procedures. Nonetheless, since many of these involve a level of qualitative human judgment, the success rate, while now quite high, is invariably less than $100 \%$.

\section{Summary}

We have described a device with moveable liquid nitrogen and liquid helium volumes that is capable of reaching over $2 \mathrm{~m}$ into a cryostat or dilution refrigerator in order to extract a polarized target of solid hydrogen deuteride (HD). This Transfer Cryostat maintains $\mathrm{HD}$ temperatures at $2 \mathrm{~K}$ and surrounds a target with a transverse
$0.1 \mathrm{~T}$ field generated by a cylindrical neodymium magnet array that is assembled in a Halbach configuration and maintained at $77 \mathrm{~K}$. Targets are engaged and disengaged using a combination of lefthanded and right-handed threads, and proper thread engagement over $2 \mathrm{~m}$ is ensured by a pivoting $2 \mathrm{~K}$ flexible connection. A springloaded 12-leaf shutter and an auxiliary shutter provide a hermetic 77 K-shield during transport of a target between cryostats. Direct tests with frozen-spin HD show negligible loss in either $\mathrm{H}$ - or Dpolarization during typical 50 min transfer periods. Multiple uses of this device indicate failure rates less than $5 \%$ per transfer. Because the process of polarizing an HD target and bringing it to an In-Beam Cryostat requires multiple transfers, the overall success rate for mounting an experiment with this device exceeds $75 \%$. This has allowed the successful execution of experiments at Brookhaven National Lab and at Jefferson Lab.

\section{Acknowledgments}

The authors are indebted to Richard Ruggiero for his help with the TC design at BNL and to the staff of the Central Institute for Engineering, Electronics and Analysis (ZEA) at Forschungszentrum Jülich for the careful construction of much of the Transfer Cryostat. We are grateful to Bill Clemens for his help e-beam welding critical components at JLab. We thank Doug Tilles and the JLab Hall-B technical crew for their dedicated assistance during the polarized target operations that utilized the Transfer Cryostat at JLab.

This work has been supported by the United States Department of Energy, Office of Nuclear Physics Division, under contract DE-AC02-98-CH10886 supporting Brookhaven National Laboratory, and under contract DE-AC05-06OR23177 under which Jefferson Science Associates operates Jefferson Laboratory, the US National Science Foundation, the German Ministries for Science and Education supporting Forschungszentrum Jülich, and the Istituto Nazionale di Fisica Nucleare of Italy.

\section{References}

[1] S. Capstick, W. Roberts, Phys. Rev. D 58 (1998) 074011.

[2] R.G. Edwards, J.J. Dudek, D.G. Richards, S.J. Wallace, Phys. Rev. D 84 (2011) 074508.

[3] A.M. Sandorfi, J. Phys. Conf. 424 (2013) 012001.

[4] A.M. Sandorfi, S. Hoblit, H. Kamano, T.-S.H. Lee, J. Phys. G 38 (2011) 053001.

[5] S. Hoblit, et al., LEGS-Spin Collaboration, Phys. Rev. Lett. 102 (2009) 172002.

[6] 〈http://www.jlab.org/exp_prog/proposals/06/PR06-101.pdf〉.

[7] N. Alexander, J. Barden, Q. Fan, A. Honig, Rev. Sci. Instrum. 62 (1991) 2729.

[8] M. Rigney, et al., Nucl. Instrum. Methods A 356 (1995) 116.

[9] X. Wei, et al., Nucl. Instrum. Methods A 526 (2004) 157.

[10] C. Thorn, A. Caracappa, in: AIP Conference Proceedings, vol. 980, 2008, p. 397.

[11] R.W. Shaw, D.E. Mapother, D.C. Hopkins, Phys. Rev. 120 (1960) 88.

[12] K. Halbach, Nucl. Instrum. Methods A 169 (1980) 1.

[13] Tridelta Magnetsysteme GmbH, D-44287 Dortmund, Germany 〈http://www. tridelta.de).

[14] Hirst Magnetic Instruments Ltd., Falmouth, Cornwall TR11-4SN, UK 〈http:// www.hirst-magnetics.com>.

[15] K.J. Strnat, D. Li, H. Mildrum, in: Proceedings of the 8th International Workshop on Rare Earth Magnets and their Applications, Dayton, OH, 6-8 May 1985, Paper no. VIII-8.

[16] American Magnetics, 112 Flint Rd, Oak Ridge, TN 37830, USA.

[17] LakeShore Cryotronics, 575 McCorkle Blvd, Westerville, OH 43082 USA

[18] Stanford Research Systems, 1290-D Reamwood Av, Sunnyvale, CA 94089, USA. 